To appear in the Astrophysical Journal

Preprint typeset using $\mathrm{LAT}_{\mathrm{EX}}$ style emulateapj v. 14/09/00

\title{
OPTICAL SPECTRAL MONITORING OF XTE J1118+480 IN OUTBURST: EVIDENCE FOR A PRECESSING ACCRETION DISK?
}

\author{
M. A. P. Torres ${ }^{1}$, P. J. Callanan ${ }^{1}$, M. R. Garcia ${ }^{2}$, J. E. McClintock ${ }^{2}$, P. Garnavich ${ }^{3}, Z_{\text {. }}$

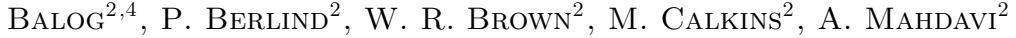 \\ To appear in the Astrophysical Journal
}

\begin{abstract}
We present spectroscopic observations of the X-ray transient XTE J1118+480 acquired during different epochs following the 2000 March outburst. We find that the emission line profiles show variations in their double-peak structure on time scales longer than the $4.1 \mathrm{hr}$ orbital period. We suggest that these changes are due to a tidally driven precessing disk. Doppler imaging of the more intense Balmer lines and the HeII $\lambda 4686$ line shows evidence of a persistent region of enhanced intensity superposed on the disk which is probably associated with the gas stream, the hotspot or both. We discuss the possible origins of the optical flux in the system and conclude that it may be due to a viscously heated disk.
\end{abstract}

Subject headings: accretion, accretion disks — binaries: close — stars: individual: XTE J1118+480 X-rays: stars

\section{INTRODUCTION}

X-ray Novae (XRNe) form a subclass of Low Mass Xray binaries (LMXBs) which has provided us with some of the best stellar-mass black hole candidates (see the reviews by van Paradijs \& McClintock 1995, van Paradijs 1998 and Charles 1998). These systems undergo occasional outbursts, with a recurrence time scale of decades, caused by an episode of intense mass transfer onto the compact primary via an accretion disk. A disk instability (Cannizzo 1993, 1998) is generally invoked as the triggering mechanism for the outburst.

The XRN XTE J1118+480 was discovered on 2000 March 29 by the Rossi X-Ray Timing Explorer All-Sky Monitor (RXTE ASM) at the beginning of a prolonged outburst (Remillard et al. 2000). Reanalysis of the ASM data showed a previous outburst episode during January 2-29 (MJDs 51,545 - 51,572; see Figure 1). The optical counterpart was identified with a 12.9 magnitude star coincident with an 18.8 magnitude object in the USNO catalogues (Uemura, Kato \& Yamaoka 2000a). Uemura (2000b) claimed the detection of superhumps in the outburst light curve with a periodicity of $0.1709 \mathrm{~d}$. The spectrum of the optical counterpart was typical of X-ray binaries in outburst (Garcia et al. 2000), but the X-ray flux was low for an X-ray transient (Remillard et al. 2000). A high inclination system (Garcia et al. 2000) and/or a 'minioutburst' state (Hynes et al. 2000) have been suggested to explain the anomalous X-ray flux. Dubus et al. (2001) presented the first constraints on the system parameters from the analysis of spectroscopic observations during the outburst. McClintock et al. $(2000,2001 \mathrm{a})$ and Wagner et al. $(2000,2001)$ derived an orbital period that was shorter than the Uemura's determination (by $0.4-0.6 \%$ respectively) from radial velocity measurements near quiescence. These authors determined the mass of the primary star to be $>6 M_{\odot}$. This places XTE J1118+480 among the dynamically confirmed black holes.

In this paper we present the results from a systematic spectroscopic campaign. Data were collected on 21 nights during 2000 March-July; on four of these nights we obtained almost complete orbital phase coverage.

\section{OBSERVATIONS AND DATA REDUCTION}

We monitored XTE J1118+480 for more than three months, beginning 2000 March 31 (UT) and continuing until July 10. The VSNET light curve, which covers most of this period, shows that the source brightness was $\mathrm{V}=13 \pm 0.3$ during 2000 April 3 - June 6. Optical spectra were obtained using the FAST spectrograph (Fabricant et al. 1998) attached to the $1.5 \mathrm{~m}$ Tillinghast telescope of the Fred Lawrence Whipple Observatory. The spectra cover the 3600 - $7500 \AA$ range with a resolution of $4.5 \AA$ FWHM. A HeNeAr lamp spectrum was taken inmediately after each object exposure in order to determine the wavelength calibration. The flux standards Feige 34, Feige 56, Hiltner 600, and BD+33 2642 were observed throughout the run in order to correct for the instrumental response. While occasional clouds and variable seeing do not allow an absolute flux calibration, the shape of the continuum is reliable except at the extreme blue and red ends (shortward of $4000 \AA$ and longward of $6800 \AA$ ). Spectra were extracted, wavelength- and flux-calibrated using the KPNO IRAF package. Details of the observations are summarized in Table 1.

\section{ANALYSIS}

\subsection{The Averaged Spectrum}

In Figure 2, we present the spectrum of XTE J1118+480 obtained by averaging the individual spectra observed on night March 31. The spectrum shows clearly the presence

${ }^{1}$ Physics Department, University College, Cork, Ireland; mapt@phys.ucc.ie, paulc@ucc.ie

2 Harvard-Smithsonian Center for Astrophysics, 60 Garden St, Cambridge, MA 02138; mgarcia@cfa.harvard.edu, jem@cfa.harvard.edu, pzhao@cfa.harvard.edu, pberlind@cfa.harvard.edu, wbrown@cfa.harvard.edu, mcalkins@cfa.harvard.edu, amahdavi@cfa.harvard.edu

${ }^{3}$ Department of Physics. University of Notre Dame, 213 Nieuwland Science Hall, Notre Dame, Indiana 46556-5670; pgarnavi@nd.edu

${ }^{4}$ on leave from Dept of Optics and Quantum Electronics, University of Szeged, Dom ter 9., H-6720 Szeged, Hungary 
of broad double-peaked emission lines of HeII $\lambda 4686$ and the Balmer series (up to $\mathrm{H} \delta$ ). Weaker HeII $\lambda 5412$ and emission lines from HeI at $\lambda \lambda 4471,4921,5875$, and 6678 are recognizable. Except for $\mathrm{H} \alpha$, the emission cores in the Balmer lines and HeI $\lambda 4471$ are contained within broad absorption features. The Bowen blend $\lambda \lambda 4640-4650$ is not present during this night (but see below). The broad bump at $4200 \AA$ is probably an instrumental artifact. The main interstellar feature is the faint blend due to the $\mathrm{Na}$ D doublet at 5890 and $5896 \AA$. We measure the equivalent width of this feature to be $\sim 0.02 \AA$ (close to the noise level) indicating very low interstellar absorption. However, we cannot exclude a contribution from the HeI $\lambda 5875$ emission line and consequently the measured equivalent width is an upper limit. Longward of $\sim 6800 \AA$, the spectra are contaminated by telluric features.

As reported by Garcia et al. (2000), two unusual absorption features at 6479 and $6516 \AA$ were present on the blueward side of the $\mathrm{H} \alpha$ profile in all our observations (see Figures 3 and 4 ). They must be associated with the accretion disk because in outburst the disk spectrum strongly dominates over the spectrum of the faint $(\mathrm{V}=18.8)$ secondary star. We suggest that both features are due to the superposition of a single broad absorption trough and an emission component due to a blend of FeI/CaI lines. This metallic blend is observed in absorption at $\lambda 6495$ in late G-K stars (Horne, Wade \& Szkody 1986), which implies that it originates in regions of the disk with $\mathrm{T}_{\text {eff }} \sim 4200$ $6000 \mathrm{~K}$. It is also possible that FeII $\lambda 6516$ contributes to the absorption features at this wavelength.

The spectra acquired during the same night or contigous nights (when the number of spectra was limited) were averaged. The resulting mean spectra were de-reddened using $E(B-V)=0.024$ and $A_{V} / E(B-V)=3.1$. The colour excess was estimated by using the hydrogen column in the line of sight $\left(\mathrm{N}_{H}=1.34 \times 10^{20} \mathrm{~cm}^{-2}\right.$; Dickey \& Lockman 1990) and the relation between $\mathrm{N}_{H}$ and $E(B-V)$ of Bohlin, Savage \& Drake (1978). Next we fitted the dereddened spectra with a power law of the form $F_{\lambda} \propto \lambda^{\alpha}$ after masking the major emission lines. We measure a mean power law index of $\alpha=-2.5 \pm 0.1$ in agreement with the values reported by Dubus et al. (2001).

\subsection{The Long-term Evolution of the Emission Lines}

We studied the more intense emission lines as follows: for each spectrum we fitted a low order spline to the adjacent continuum of every profile after masking the lines, and the spectrum was divided by the fitted function. Next, for those nights during which we obtained more than two spectra, we averaged the spectra. The $\mathrm{H} \alpha$ and HeII $\lambda 4686$ line profiles were fitted with a 2-gaussian function and the $\mathrm{H} \beta$ profile with a 3-gaussian function (with one used to account for the absorption component) using the Marquardt algorithm (Bevington 1969). Tables 2 and 3 list the values of the main fitted line parameters for the nights with good orbital coverage. In Table 4 we give the measured full width zero intensity (FWZI) and equivalent widths (EWs) for $\mathrm{H} \alpha, \mathrm{H} \beta$, and HeII $\lambda 4686$. The EWs for additional lines are presented in Table 5. The uncertainties in the EWs and FWZIs were estimated by looking at the scatter in the values when selecting different wavelength intervals to set the local continuum level.
The mean peak-to-peak velocity separation for the $\mathrm{H} \alpha$ and $\mathrm{H} \beta$ lines is comparable $\left(\sim 1200 \mathrm{~km} \mathrm{~s}^{-1}\right)$ with little change between different epochs. The peak-to-peak velocity separation of the HeII $\lambda 4686$ line ranges from 1600 to $2000 \mathrm{~km} \mathrm{~s}^{-1}$. This result suggests that the emission at $\mathrm{H} \alpha$ and $\mathrm{H} \beta$ arises at a similar distance from the compact object and the HeII $\lambda 4686$ line is emitted from regions closer to the compact object. The velocity separation of the double-peaks in the $\mathrm{H} \alpha$ line is consistent with the values reported for other black hole XRNe during quiescence and outburst (see e.g. Table 1 in Smith, Filippenko \& Leonard 1999). The FWZI of the $\mathrm{H} \beta$ absorption component implies a projected velocity of $\sim 2900 \mathrm{~km} \mathrm{~s}^{-1}$ for the inner part of the optically-thick accretion disk emitting at this wavelength.

The most remarkable feature in the evolution of the emission lines is the change in the double-peak intensity, from almost symmetric peaks to enhanced redshifted or blueshifted peaks (see Figure 3). Even though this behavior in the poorly sampled nights might be attributable to an S-wave, a different explanation is required for the symmetric/asymmetric averaged profiles on March 31, April 12, 29, and May 25, where uniform orbital phase coverage should ensure the cancellation of any S-wave effect in the averaged profiles.

Finally, the Bowen blend appears marginally on April 3, probably blended with HeII $\lambda 4686$ on April 12 and clearly enhanced on April 29. A glance at the RXTE/ASM light curve (1.3-12.2 keV) of XTE J1118+480 (see Figure 1) shows that the enhancement in intensity occurs near the maximum in X-ray flux. On May 25, during the slow decay of the X-ray outburst, both the Bowen Blend and HeII $\lambda 4686$ have decreased in intensity.

\section{DOPPLER TOMOGRAPHY}

Although the emission lines show variations in the double-peak structure due to an S-wave emission component, our attempts to find a periodic radial velocity modulation failed due mainly to our low spectral resolution and the complexity of the line profiles. Fortunately, we can obtain valuable information by using the Doppler Tomography technique (Marsh \& Horne 1988) on those data sets with good orbital phase coverage. This technique reconstructs the brightness distribution of the binary system in velocity space, allowing us to localize the emission structures which are not easily recognizable in the individual spectra.

We use the maximum-entropy method (MEM) of building the tomograms, which gradually builds the emission structure from a default uniform image by reducing the $\chi^{2}$ between the data and the model fit. The optimal solution is selected among infinite possibilities by maximizing the entropy of the image. To obtain the tomograms, the orbital phases were determined using an orbital period $\mathrm{P}_{\text {orb }}=0.169937 \pm 0.000007 \mathrm{~d}$ and a time of minimum light $\mathrm{T}_{o}(\mathrm{HJD})=2,451,880.1060 \pm 0.0011^{5}$, which corresponds 
to closest approach of the secondary to the observer.

We have computed Doppler maps of the HeII $\lambda 4686$, $\mathrm{H} \alpha$, and $\mathrm{H} \beta$ lines for the nights March 31, April 12, 29, and May 25. Because MEM Doppler tomography cannot deal with negative values, the broad absorption in the $\mathrm{H} \beta$ line was removed from each spectrum through a high-order spline fit to the profile, after masking the emission core. Figures 5-8 display the derived Doppler maps: the upper panels show the spectra in the form of trailed spectrograms constructed by folding the line profile spectra into 15 phase bins. The bottom panels show the MEM tomograms. Similar results were obtained using the filtered back-projection (FBP) technique ${ }^{6}$ (see Appendix in Marsh \& Horne 1988), confirming that the $\mathrm{H} \beta$ MEM images are not affected by the removal of the broad absorption troughs.

The trailed spectra show clear changes in the line behaviour: while on March 31 the HeII $\lambda 4686$ line and, to a lesser extent, the Balmer lines show a double-peaked profile with an S-wave component (which is also detectable on April 1), only enhancement in the red/blue peak of the profile is observed during the other nights. On the other hand, two emission structures are visible in the tomograms: the first is a ring, clearly visible in the Balmer images, which is a signature of emission arising from a rotating accretion disk. The second is an intense bowshaped emission region, present both in the Balmer and HeII $\lambda 4686$ maps. Its position in the $-\mathrm{V}_{X},+\mathrm{V}_{Y}$ quadrant places the plausible origin for this emission in the accretion gas stream, in the hotspot (the stream/disk impact region) or both. The presence of some of these physical structures on different nights argues for continuous mass transfer from the companion star during our observations.

We have plotted the theoretical path of the gas stream and the Keplerian velocities of the disk along the stream for $K_{2}=698 \mathrm{~km} \mathrm{~s}^{-1}$ (where $K_{2}$ is the radial velocity semiamplitude of the companion star; McClintock et al. 2001a) and a mass ratio $q=0.07$ (a reasonable value for a latetype secondary star and a $7 \mathrm{M}_{\odot}$ compact object). The Doppler maps appear to show that the region of enhanced intensity only partially overlaps the region delineated by these trajectories. A similar departure from the theoretical paths has been observed in the CVs OY Carinae (Harlaftis \& Marsh 1996) and WZ Sge (Spruit \& Rutten 1998). Note however that the accuracy of our ephemeris is such that random offsets amounting to 0.06 in orbital phase (i.e. an angular offset of about $20^{\circ}$ ) are possible. If the maps are rotated clockwise by $\sim 20^{\circ}$, the bright spot would lie between both trajectories. Again this would be a strong indicator for the presence of a hotspot (see Marsh et al. 1990) or/and the accretion gas stream.

\section{DISCUSSION}

\subsection{Disc Precession}

Asymmetric emission line profiles have been observed in several SU UMa systems, i.e. in those dwarf novae (DNe) which suffer superoutbursts (for a review see Warner 1995). After maximum light, the SU UMa optical light curves exhibit a modulation at a period slightly longer than the binary orbital period, called the superhump period. A tidal resonance model (see e. g. Whitehurst \& King 1991) is commonly used to explain the superhump period: at this stage of the superoutburst an extended accretion disk forms which, as a consequence of the tidal influence of the secondary, becomes elliptical and precesses. It is presently believed that enhanced viscous energy dissipation due to the tidal stresses from the secondary is the dominant superhump light source. Such a disk has been used to explain, for example, the asymmetric $\mathrm{H} \alpha$ profile in OY Carinae (Hessman 1992) and the systematic variations in the velocities of the central absorption feature observed in the emission lines of ZCha (Vogt 1981; Honey et al. 1988). Theoretical line profiles obtained using a model of a precessing eccentric accretion disk suggest that this is the case for IY UMa (Wu, Li \& Gao 2001). Similarly the long term variations we observe in the line profiles of XTE J1118+480 can be explained by the presence of an eccentric disk precessing around the compact primary with a period longer than the $4.1 \mathrm{hr}$ orbital period. The detection of superhumps during the outburst (Uemura 2000b) supports this suggestion. Indeed, our May $25 \mathrm{H} \beta$ map in particular suggests an elliptical disk (see Figure 8). However, the extent of the bow-shaped emission region makes any such ellipticity very difficult to quantify.

An estimation of the precession period can be calculated using $P_{\text {prec }}=P_{\text {orb }} P_{\text {sh }} /\left(P_{\text {sh }}-P_{\text {orb }}\right)$ (see Warner 1995), where $\mathrm{P}_{\text {prec }}$ and $\mathrm{P}_{s h}$ are the precession and superhump periods respectively. The values of $\mathrm{P}_{s h}$ reported by Uemura (2000b) and $\mathrm{P}_{\text {orb }}(\S 4)$ imply a precession period of $\sim 30-80 \mathrm{~d}$. A precession period near quiescence of about $52 \mathrm{~d}$ was estimated by Casares et al. (2001). Since the motion of the compact object is expected to be small $\left(\mathrm{K}_{1}=\mathrm{qK}_{2}=49 \mathrm{~km} \mathrm{~s}^{-1}\right)$, we searched for a long term periodic modulation in the centroids of the HeII $\lambda 4686$ line profiles shown in Figure 3. We focused on this line because its measurement was unaffected by any nearby absorption feature. The centroid of the profiles was obtained by fitting a gaussian after masking the core of the line. A period search did not show any significant modulation within the expected period range. In addition, we searched the red/blue peak flux ratio of the HeII line for variability consistent with the predicted precession period range, but also without success. This perhaps is not surprising as we have only four nights of uniform orbital coverage.

\subsection{X-ray Heating vs Viscous Dissipation}

Does X-ray heating generate a significant amount of the optical flux from the disk? The slope of the optical spectrum might provide an answer, as for outbursting (optically thick) disks, standard disk theory predicts $F_{\lambda} \propto \lambda^{-2.33}$ if there is no X-ray heating (Beall et al. 1984). However, the wide range of power law indices $(-1<\alpha<-3)$ measured by Shahbaz et al. (1996) in their sample of LMXBs indicates that the slope of the optical spectrum alone does not seem to be a reliable indicator of X-ray heating. On the other hand, the observation of su-

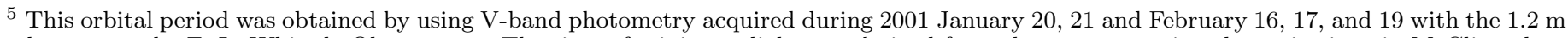

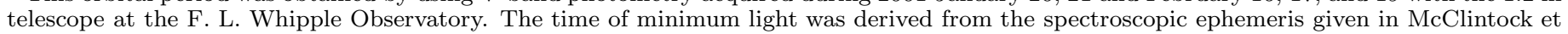
al. (2001a)

6 This technique has the advantage that no treatment is necessary for negative values in the spectra. 
perhumps in XTE J1118+480 and other black hole XRNe during outburst (O'Donoghue \& Charles 1996) does not in itself exclude X-ray heating as the dominant source of optical luminosity (Haswell et al. 2001).

Another way to estimate the contribution from X-ray irradiation is to use the relation between the absolute magnitude and the orbital period/X-ray luminosity for LMXBs found by van Paradijs \& McClintock (1994). Wagner et al. (2001) derive a distance of $\mathrm{d}=1.9 \pm 0.4 \mathrm{kpc}$ for XTE J1118+480. This implies an absolute magnitude of $\mathrm{M}_{V}=1.6_{-0.4}^{+0.5}$ in disagreement with the expected value for an irradiation-dominated disk of $\mathrm{M}_{V} \approx 3.0 \pm 0.5$ (van Paradijs \& McClintock 1994, Fig. 2). Here we have corrected the resultant absolute magnitude by $-0.7 \mathrm{mag}$ to take into account that the visual luminosity due to X-ray reprocessing in the disk scales with the compact object mass as $\sim \mathrm{M}_{1}{ }^{1 / 3}$. A $7 \mathrm{M}_{\odot}$ black hole was assumed. Furthermore, it is clear from Fig. 2 of Uemura (2000c) that on at least two occasions, the source of the optical flux was other than X-ray irradiation. The first, before the end of the January outburst, was an optical flare (of amplitude $\gtrsim 1.5 \mathrm{mag}$ ) without any accompanying X-ray flare. In the second event, at the beginning of the March outburst, the steep rise in the optical flux was accompanied by a much slower increase in the X-ray flux. Hence we think it unlikely that X-ray reprocessing dominates the outburst optical flux.

Viscous dissipation in the disk is an alternative explanation of the optical flux. Using the $\mathrm{M}_{V}-\mathrm{P}_{\text {orb }}$ relation for $\mathrm{DNe}$ in outburst (Warner 1987), we have estimated $\mathrm{M}_{V}=4.7$ for a DN with an orbital period of $4.1 \mathrm{hr}$. But since the area of the disk scales with the primary mass as $\mathrm{M}_{1}{ }^{2 / 3}$ for a fixed orbital period (Cannizzo 1998) and the energy released at a given distance from the primary scales with the primary mass as $\mathrm{M}_{1}$ (Frank, King \& Raine 1985), we expect the optical flux generated by a disk in a black hole XRN to be $\sim \mathrm{M}_{1}{ }^{5 / 3}$ higher than that emitted by a disk surrounding the $\sim 1 \mathrm{M}_{\odot}$ primary in a DN. This correction implies $\mathrm{M}_{V} \sim 1.2$ if XTE J1118+480 contains a $7 \mathrm{M}_{\odot}$ black hole. Note that we have assumed a moderate inclination for the binary system. For an inclination $i=70^{\circ}$, the outburst absolute magnitude of the viscously heated disk corrected using the prescription of Warner (1987) is $\mathrm{M}_{V}^{\text {corr }} \sim 1.9$, close to the observed absolute magnitude. For $i \simeq 80^{\circ}$, the inclination favoured when fitting the near quiescence optical light curve with an ellipsoidal model (McClintock et al. 2001a; Wagner et al. 2001), the above correction gives $\mathrm{M}_{V}^{\text {corr }} \sim 2.8$. Although this magnitude is fainter than the observed absolute magnitude, DNe superoutbursts are 0.5-1 magnitudes brighter than normal outbursts and even 2 magnitudes brighter (or more) in the case of the TOAD superoutbursts (Howell, Szkody \& Cannizzo 1995; Kuulkers, Howell \& van Paradijs 1996). If the outburst in XTE J1118+480 is similar to a DN superoutburst then $\mathrm{M}_{V}^{\text {corr }} \sim 1.8$. The presence of a bright, viscously heated disk is supported by the detection of a significant Balmer jump in the ultraviolet spectrum (Haswell, Hynes \& King 2000; McClintock et al. 2001b), which suggests an appreciable thermal disk contribution to the emission. Hence the viscously heated disk is likely to be a significant contributor to the optical flux during the outburst of XTE J1118+480 as in the SU UMa systems.

\section{CONCLUSIONS}

Multi-epoch optical spectroscopic observations of XTE J1118+480 yield valuable information about the properties and evolution of the accretion disk during outburst. The data reveal strong double-peaked $\mathrm{H} \alpha, \mathrm{H} \beta$, and HeII $\lambda 4686$ emission lines whose double-peak intensity varies on time scales longer than the $4.1 \mathrm{hr}$ orbital cycle. These changes in the line profiles can be interpreted as resulting from a precessing eccentric disk around the compact primary. However, a search for periodic variability in the HeII $\lambda 4686$ emission line failed to find a modulation within the estimated precession period range. Therefore conclusive evidence for a precessing disk in the system is still required. Doppler tomograms display a bright bow-shaped emission in the $-\mathrm{V}_{X},+\mathrm{V}_{Y}$ quadrant which we interpret as emission from the accretion gas stream, the hotspot or both. A more accurate ephemeris is needed to properly constrain the location of the emission within the binary system. A comparison with DNe in superoutburst shows that viscous dissipation in the disk may make a significant contribution to the optical luminosity during outburst.

Use of MOLLY, DOPPLER and TRAILER routines developed largely by T. R. Marsh is acknowledged. We thank Ann Esin for helpful comments and Makoto Uemura for providing the VSNET light curve. We are grateful to the anonymous referee for useful comments which improved the quality of the manuscript. MRG was supported by NASA contract NAS8-39073. 


\section{REFERENCES}

Beall, J. H., Knight, F. K., Smith, H. A., \& Wood, K. S. 1984, ApJ, 284,745

Bevington, P. R. 1969, Data Reduction and Error Analysis for the Physical Sciences (New York: McGraw-Hill)

Bohlin, R. C., Savage, B. D., \& Drake, J. F. 1978, ApJ, 224, 132

Cannizzo, J. 1993, in Accretion Disks in Compact Stellar Systems, ed. J. Wheeler (Singapore: World Scientific), 6

Cannizzo, J. K. 1998, ApJ, 494, 366

Casares, J., et al. 2001, IAU Circ. 7617

Charles P. A., 1998, in Theory of Black Hole Accre tion Disks, ed. M. A. Abramowicz, G. Bjornsson, \& J. E. Pringle (Cambridge: Cam bridge Univ. Press), 1

Dickey, J. M., \& Lockman, F. J. 1990, ARA\&A, 28, 215

Dubus, G., Kim Rita, S.J., Menou, K., Szkody, P., \& Bowen, D. V. 2001. ApJ, 553, 307

Fabricant, D., Cheimets, P., Caldwell, N., \& Geary, J. 1998, PASP, 110,79

Frank, J., King, A. R., \& Raine, D. J., 1985, Accretion Power in Astrophysics (Cambridge: Cambridge Univ. Press)

Garcia, M., Brown, W., Pahre, M., McClintock, J., Callanan, P., \& Garnavich, P. 2000, IAU Circ. 7392

Harlaftis, E. T., \& Marsh T. R. 1996, A\&A, 308, 97

Haswell, C. A., Hynes, R. I., \& King, A. R. 2000, IAU Circ. 7407

Haswell, C. A., King, A. R., Murray, J. R., \& Charles, P. A. 2001, MNRAS, 321, 475

Hessman, F. V., Mantel, K. H., Barwig, H., \& Schoembs, R. 1992, A\&A, 263, 147

Honey, W. B., Charles, P. A., Whitehurst, R., Barrett, P. E., \& Smale, A. P. 1988, MNRAS, 231, 1

Horne, K. \& Cook, M. C. 1985, MNRAS, 214, 307

Horne, K., Wade, R. A., \& Szkody, P. 1986, MNRAS, 219, 791

Howell, S. B., Szkody, P., \& Cannizzo, J. 1995, ApJ, 439, 337

Hynes, R. I., Mauche, C. W., Haswell, C. A., Shrader, C. R., Cui, W., \& Chaty, S. 2000, ApJ, 539, L37

Kuulkers, E., Howell, S. B., \& van Paradijs, J. 1996, ApJ, 462, L87
Marsh, T.R., \& Horne, K. 1988, MNRAS, 235, 269

Marsh, T.R., Horne, K., Schlegel, E. M., Honeycutt, R. K., \& Kaitchuck, R. H. 1990, ApJ, 364, 637

McClintock, J, Garcia, M., Zhao, P., Caldwell, N., \& Falco, E. 2000. IAU Circ. 7542

McClintock, J. E., Garcia, M. R., Caldwell, N., Falco, E. E., Garnavich, P. M., \& Zhao, P. 2001a, ApJ, 551, 147L

McClintock, J. E. et al. 2001b, ApJ, 555, 477

O'Donoghue, D., \& Charles, P. A. 1996, MNRAS, 282, 191

Remillard, R., Morgan, E., Smith, D., \& Smith, E. 2000, IAU Circ. 7389

Shahbaz, T. Smale, A. P., Naylor, T. Charles, P. A., van Paradijs, J., Hassall, B. J. M., \& Callanan, P. 1996, MNRAS, 282, 1437

Smith, I. A., Filippenko, A. V., \& Leonard, D. C. 1999, ApJ, 519, 779

Spruit, H. C., \& Rutten, G. M. 1998, MNRAS, 299, 768

Uemura, M., Kato, T., \& Yamaoka, H. 2000a, IAU Circ. 7390

Uemura, M., et al. 2000b, IAU Circ. 7418

Uemura, M., et al. 2000c, PASJ, 52, 15L

van Paradijs, J., \& McClintock, J. E. 1994, A\&A, 290, 133

van Paradijs, J., \& McClintock, J. E. 1995, in X-ray Binaries, ed. W. H. G. Lewin, J. van Paradijs \& E. P. J. van den Heuvel. (Cambridge: Cambridge Univ. Press), 107

van Paradijs, J. 1998, in The many Faces of Neutron Stars, ed. R. Buccheri, J. van Paradijs \& M. A. Alpar (Dordrecht: Kluwer), 279

Vogt, N. 1981, ApJ, 252, 653

Wagner, R. M., Foltz, C. B., Starrfield, S. G., \& Hewett, P. 2000, IAU Circ. 7542

Wagner, R. M., Foltz, C. B., Shahbaz, T., Casares, J., Charles, P. A., Starrfield, S. G., \& Hewett, P. 2001, ApJ, 556, 42

Warner, B. 1987, MNRAS, 227, 23

Warner, B. 1995, Cataclysmic Variables (Cambridge: Cambridge Univ. Press)

Whitehurst, R., \& King, A. J. 1991, MNRAS, 249, 25

Wu, X., Li, Z., \& Gao, W. 2001. ApJ, 549, L81 


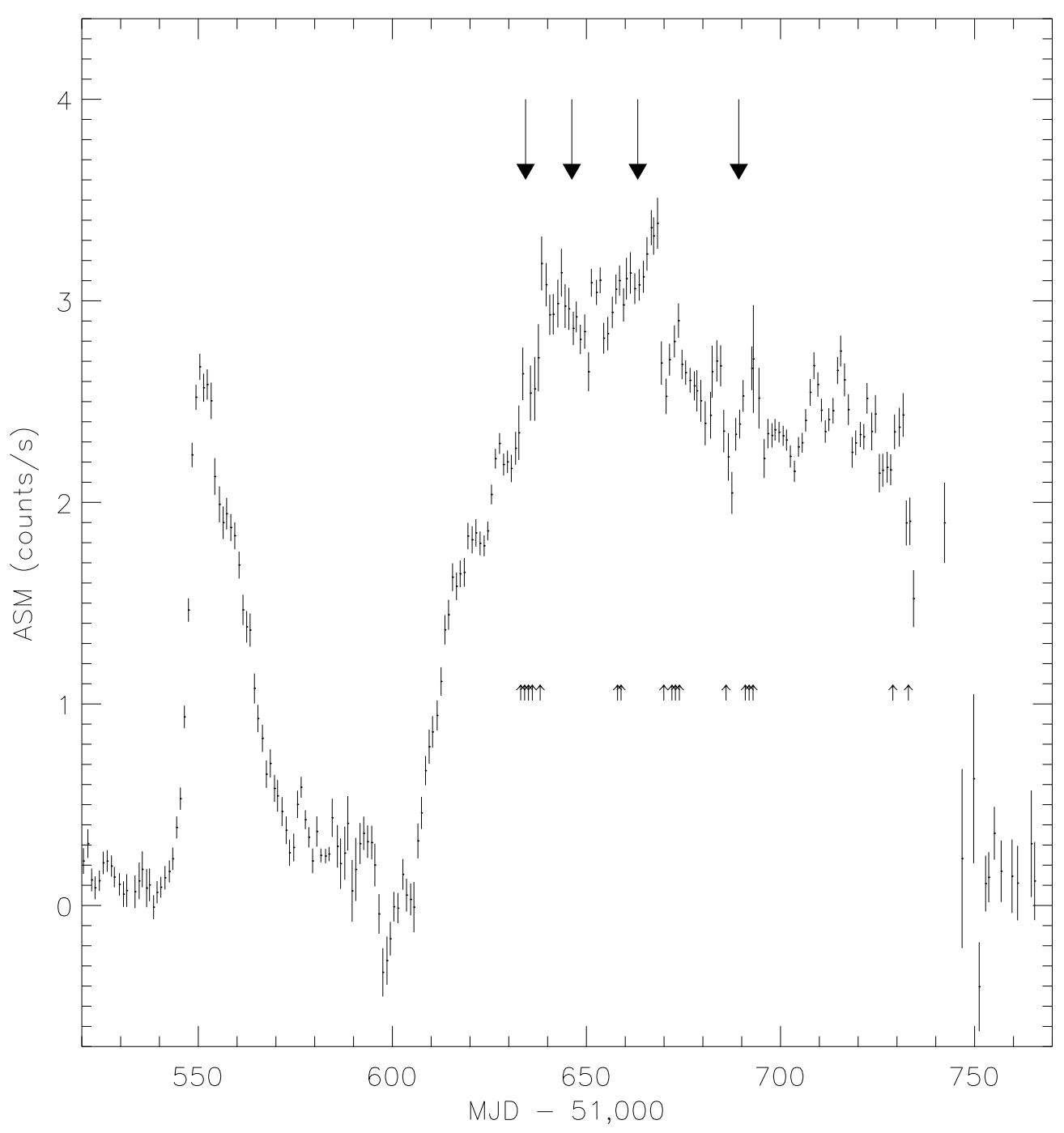

FIG. 1.- RXTE ASM outburst light curve of XTE J11184+480 after smoothing with a 3 point weighted average. The arrows indicate the dates of spectroscopic observations: long solid arrows mark the nights during which the orbital phase coverage allowed us to carry out Doppler tomography (see Table 1). 

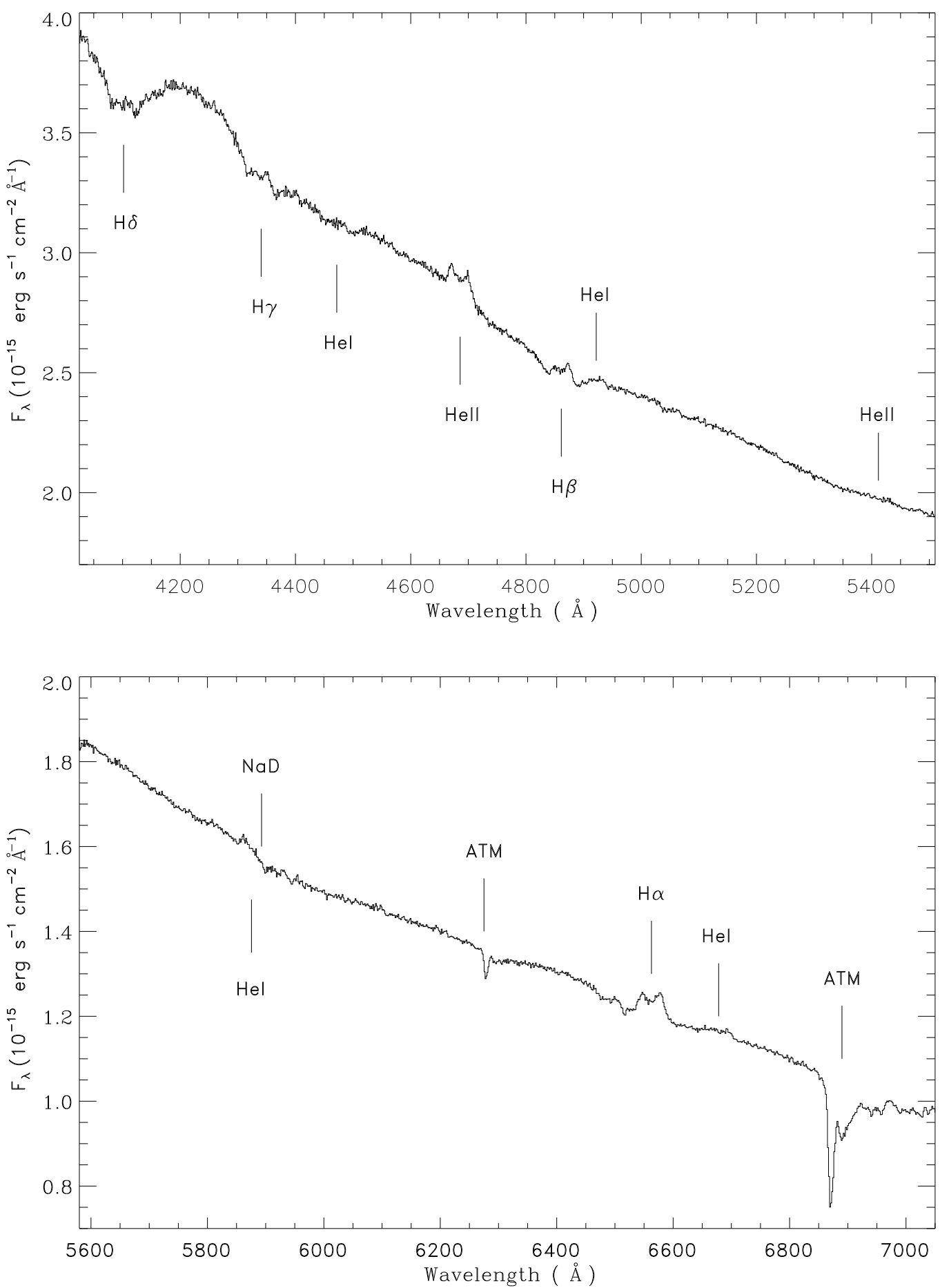

FIG. 2.- Average optical FAST spectrum of XTE J1118+480 on March 31 (UT). Major features are identified. ATM denotes the atmospheric features. 

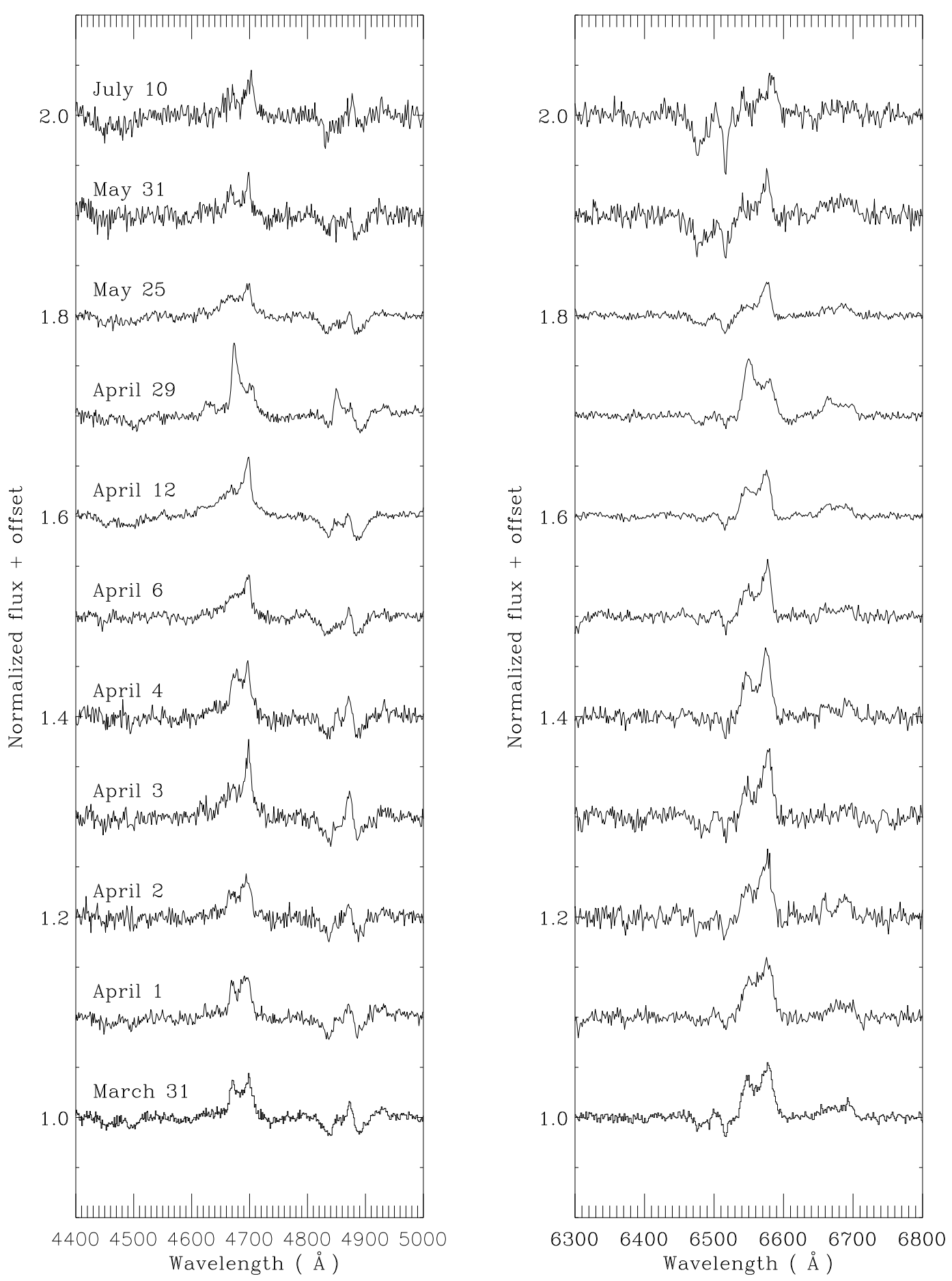

FIG. 3. - Evolution of the averaged red and blue spectrum during outburst. Individual spectra have been averaged on a night by night basis when more than two spectra were available. 

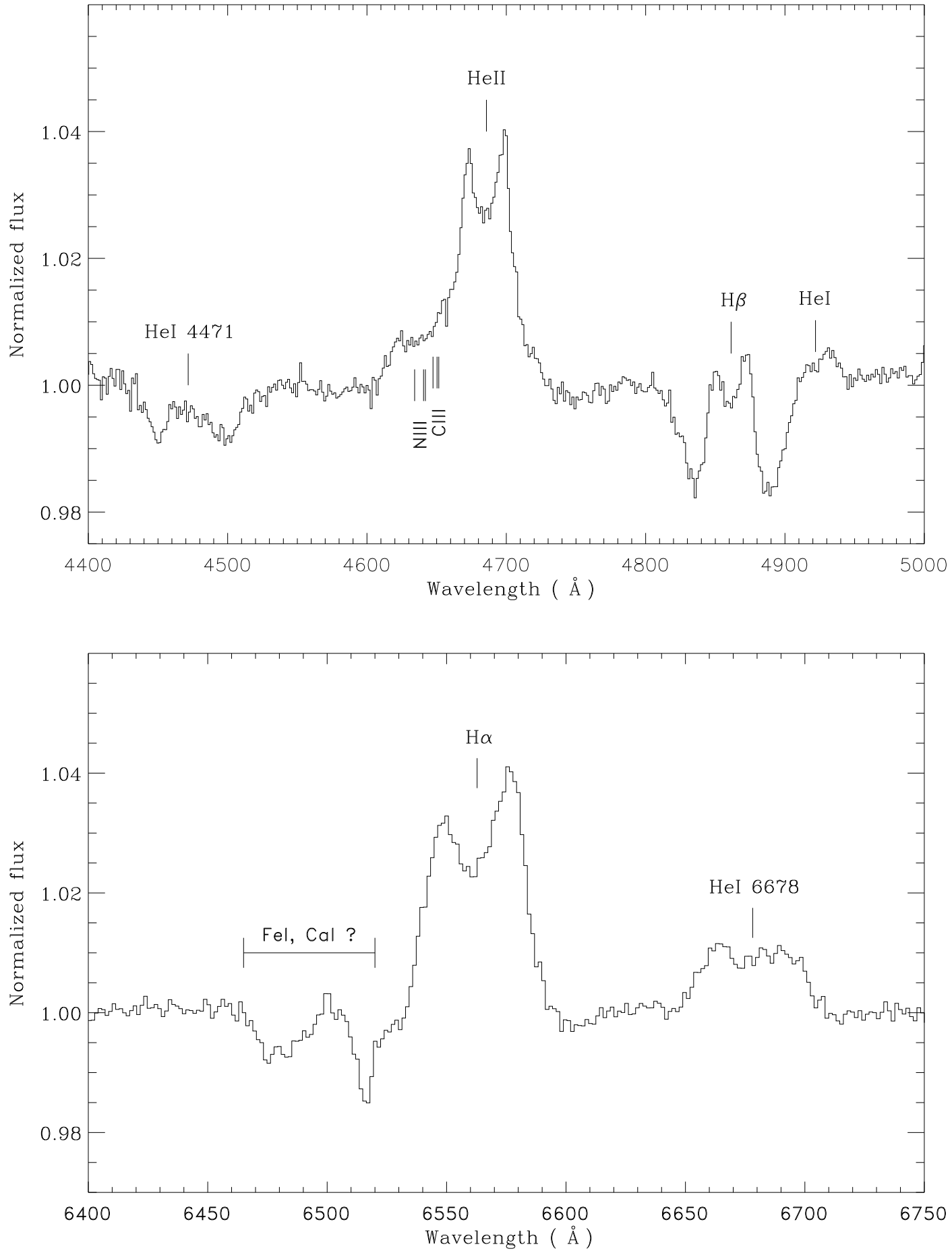

FIG. 4.- Average of the blue and red spectra of XTE J1118+480 acquired during our run. Note the two absorption features blueward H $\alpha$. 
00 March 31

$\mathrm{H} \alpha \quad \mathrm{H} \beta \quad$ HeII 4686

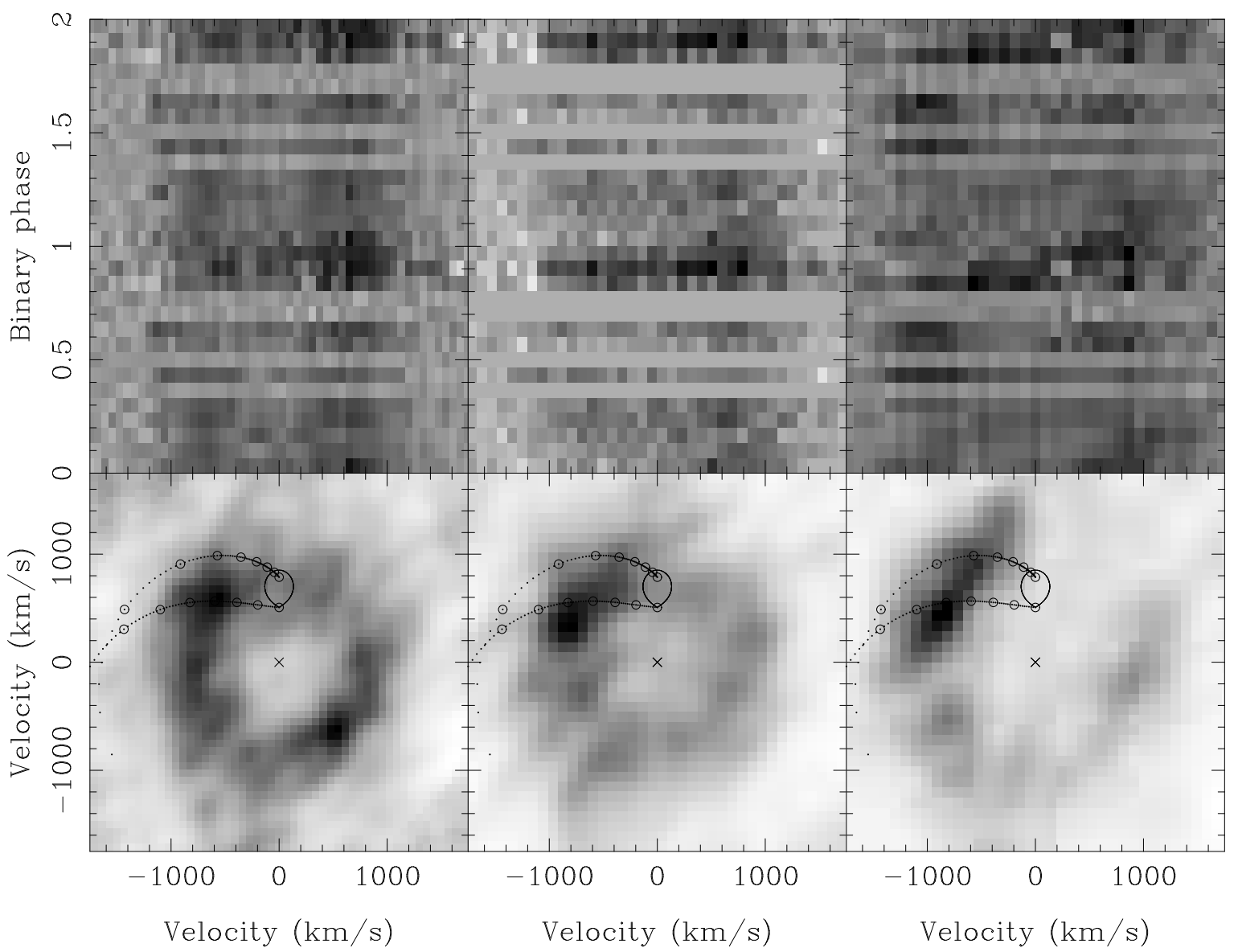

FIG. 5.- Top panels present from left to right the trailed spectrograms of $\mathrm{H} \alpha, \mathrm{H} \beta$ and HeII $\lambda 4686$. Empty strips represent gaps in the phase coverage. For the sake of clarity, the same cycle has been plotted twice. Lower panels show the computed MEM Doppler maps. The Roche lobe of the secondary star, the predicted velocities of the gas stream (lower curve) and of the disk along the stream (upper curve) are plotted for $\mathrm{K}_{2}=698 \mathrm{~km} \mathrm{~s}^{-1}$ and $q=0.07$. Distances in multiples of $0.1 \mathrm{R}_{L 1}$ are marked along both curves with open circles. The center of mass of the system is denoted by a cross. 
00 April 12

$\begin{array}{lll}\mathrm{H} \alpha & \mathrm{H} \beta & \text { HeII } 4686\end{array}$

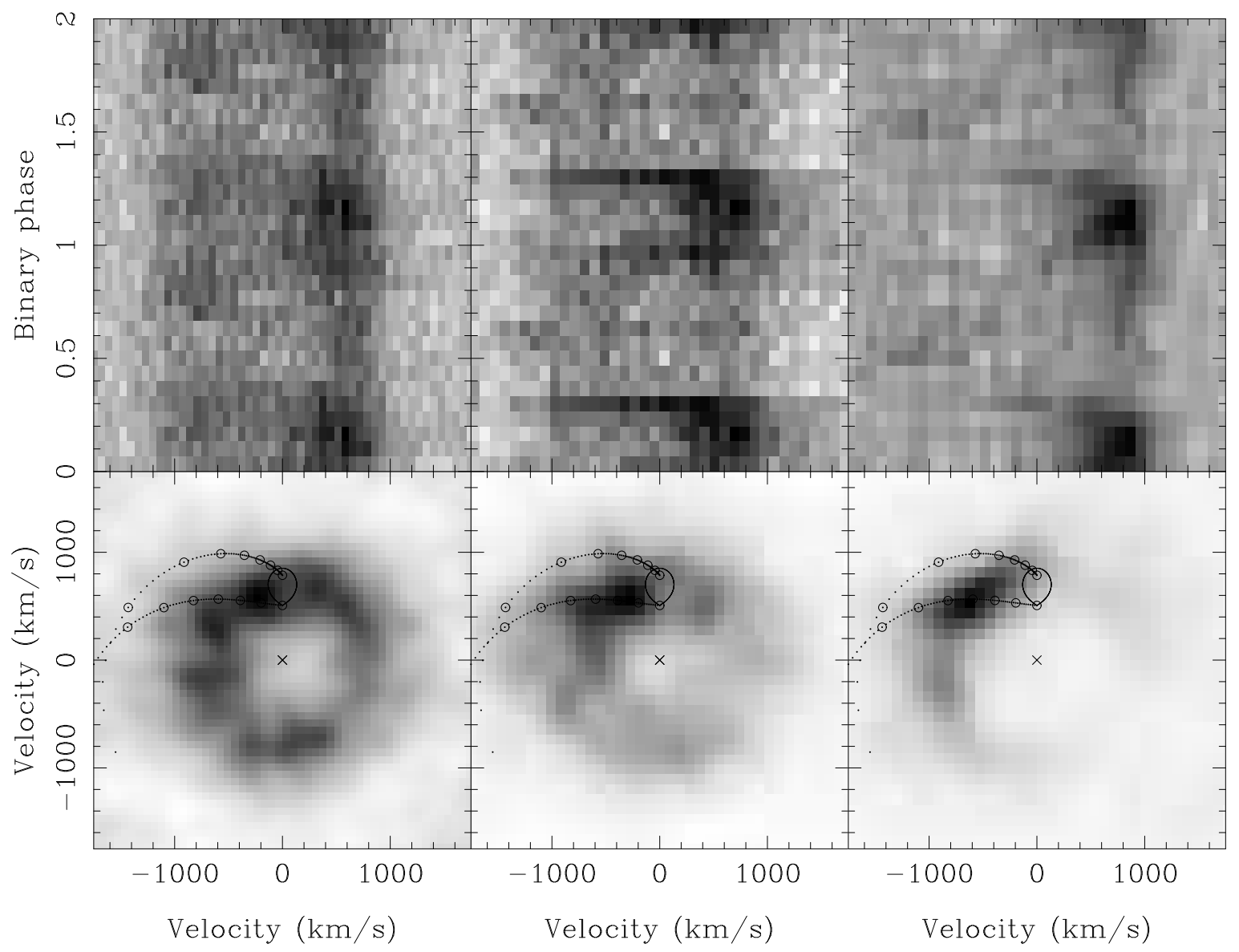

FIG. 6.- Trailed spectrogram and Doppler maps of $\mathrm{H} \alpha, \mathrm{H} \beta$ and HeII $\lambda 4686$ built with data acquired during April 12. 
00 April 29

$\mathrm{H} \alpha \mathrm{H} \beta \quad$ HeII 4686

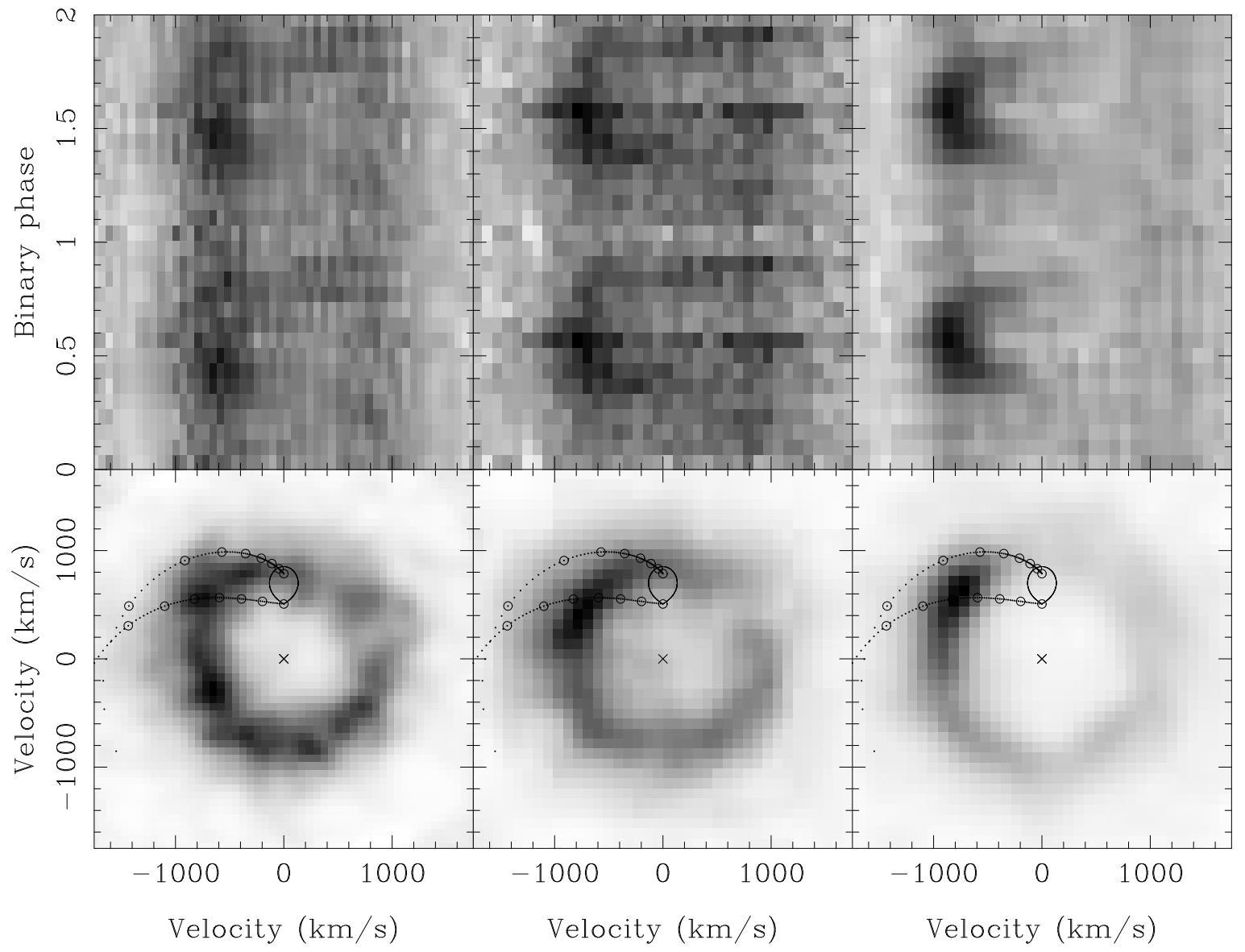

FIG. 7.- Trailed spectrogram and Doppler maps of $\mathrm{H} \alpha, \mathrm{H} \beta$ and HeII $\lambda 4686$ built with data acquired during April 29. 
00 May 25

$\begin{array}{lll}\mathrm{H} \alpha & \mathrm{H} \beta & \text { HeII } 4686\end{array}$

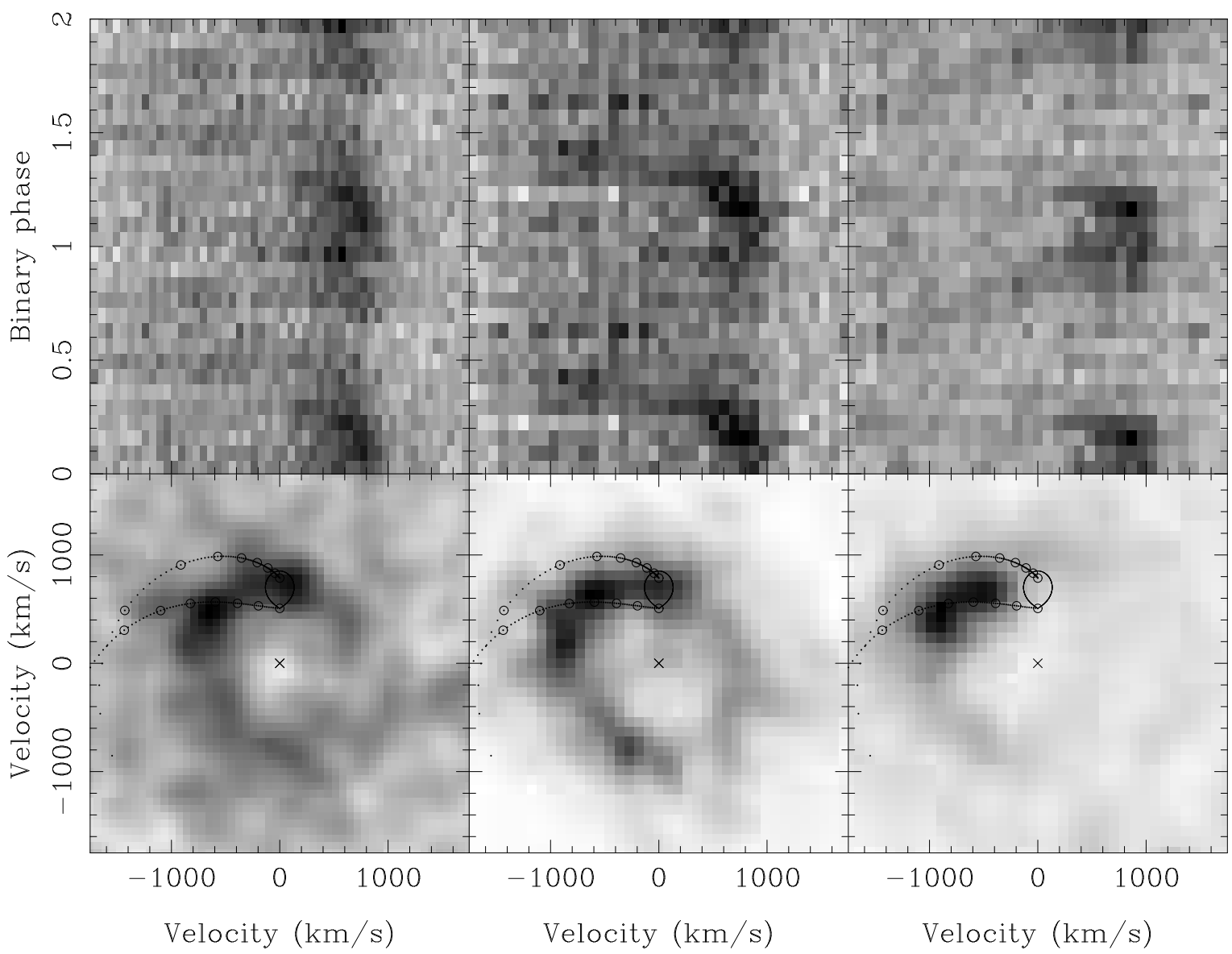

FIG. 8. - Trailed spectrogram and Doppler maps of $\mathrm{H} \alpha, \mathrm{H} \beta$ and HeII $\lambda 4686$ built with data acquired during May 25 . The $\mathrm{H} \beta$ map suggests an eccentric disk. Note that only the FBP tomogram was computed for $\mathrm{H} \alpha$ because of the proximity of the blueward absorption feature to the wing of the line (see Figure 3). 
TABLE 1

JOURNAL OF OBSERVATIONS

\begin{tabular}{|c|c|c|c|c|c|}
\hline $\begin{array}{l}\text { Date } \\
(U T)\end{array}$ & No. spectra & $\begin{array}{c}\text { Exp. time } \\
(s)\end{array}$ & $\begin{array}{c}\text { HJD start } \\
(+2,451,000 .)\end{array}$ & $\begin{array}{c}\text { HJD end } \\
(+2,451,000 .)\end{array}$ & $\begin{array}{c}\text { seeing } \\
(\operatorname{arcsec})\end{array}$ \\
\hline $00 \operatorname{March} 31^{\mathrm{a}}$ & 15 & $1 \times 300,9 \times 600,3 \times 900,2 \times 1200$ & 634.7056 & 634.9375 & $2-3$ \\
\hline 00 April 1 & 7 & 600 & 635.6384 & 635.9361 & $1-2$ \\
\hline $00 \quad, \quad 2$ & 3 &, & 636.6492 & 636.9576 &, \\
\hline $00,, \quad 3$ &, &, & 637.6169 & 637.9586 & , \\
\hline $00 \quad,, 4$ &, & , & 638.6186 & 638.9340 & , \\
\hline $00,, \quad 6$ & 7 & & 640.6394 & 640.9806 & 1 \\
\hline $00 \quad, \quad 12^{\mathrm{a}}$ & 43 & 360 & 646.6485 & 646.8406 & $1-2$ \\
\hline $00 \quad, \quad 26$ & 2 & 600 & 660.6628 & 660.8245 &, \\
\hline $00 \quad, \quad 27$ & 1 &, & 661.6482 & 661.6482 & , \\
\hline $00, \quad 29^{\mathrm{a}}$ & 35 & 480 & 663.6204 & 663.8294 & $"$ \\
\hline 00 May 8 & 1 & 300 & 672.6557 & 672.6557 & $1-2$ \\
\hline $00, \quad, \quad 10$ &, & & 674.7523 & 674.7523 &, \\
\hline $00 \quad, \quad 11$ &, & 600 & 675.6582 & 675.6582 & , \\
\hline $00 \quad, \quad 12$ & $"$ &, & 676.6679 & 676.6679 &, \\
\hline $00 \quad, \quad 24$ & . & , & 688.6886 & 688.6886 & , \\
\hline $00, \quad 25^{\mathrm{a}}$ & 44 & $40 \times 300,4 \times 600$ & 689.6596 & 689.8398 & 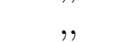 \\
\hline $00 \quad, \quad 29$ & 3 & $1 \times 60,2 \times 180$ & 693.6482 & 693.6534 & 2 \\
\hline $00 \quad, \quad 30$ &, & 300 & 694.6414 & 694.6503 & \\
\hline $00 \quad, \quad 31$ & 5 & $"$ & 695.6403 & 695.6569 & $1-2$ \\
\hline 00 July 6 & 1 & 900 & 731.6686 & 731.6686 & 1 \\
\hline $00 \quad, \quad 10$ & 8 & 300 & 735.6467 & 735.6733 & $1-2$ \\
\hline
\end{tabular}

${ }^{a}$ Orbital phase complete or nearly complete.

TABLE 2

Fits to the H $\alpha$ ANd Heil $\lambda 4686$ Line Profiles

\begin{tabular}{|c|c|c|c|c|}
\hline \multirow[b]{2}{*}{$\begin{array}{l}\text { Date } \\
U T\end{array}$} & \multicolumn{2}{|c|}{$\mathrm{H} \alpha$} & \multicolumn{2}{|c|}{ HeII $\lambda 4686$} \\
\hline & $\begin{array}{c}\mathrm{V}_{b} \\
\left(\mathrm{~km} \mathrm{~s}^{-1}\right)\end{array}$ & $\frac{\mathrm{V}_{r}}{\left(\mathrm{~km} \mathrm{~s}^{-1}\right)}$ & $\begin{array}{c}\mathrm{V}_{b} \\
\left(\mathrm{~km} \mathrm{~s}^{-1}\right)\end{array}$ & $\left(\mathrm{km} \mathrm{s}^{-1}\right)$ \\
\hline March 31 & $-643 \pm 24$ & $598 \pm 20$ & $-881 \pm 30$ & $721 \pm 30$ \\
\hline April 12 & $-601 \pm 29$ & $521 \pm 17$ & - & $707 \pm 13$ \\
\hline April 29 & $-576 \pm 14$ & $620 \pm 25$ & $-756 \pm 12$ & $1226 \pm 50$ \\
\hline May 25 & - & $578 \pm 16$ & - & $760 \pm 26$ \\
\hline
\end{tabular}

Note. - The table shows the shift respect to the rest wavelength of the line of the blue $\left(\mathrm{V}_{b}\right)$ and red $\left(\mathrm{V}_{r}\right)$ peaks (whenever they were measurable). The uncertainties were calculated after the error bars had been scaled to give $\chi_{\nu}^{2}=1$. 
TABLE 3

Fits to the H $\beta$ Line Profile

\begin{tabular}{|c|c|c|c|c|c|c|c|}
\hline \multirow[b]{2}{*}{$\begin{array}{l}\text { Date } \\
\text { UT }\end{array}$} & \multicolumn{4}{|c|}{ 3-gaussian fit } & \multirow[b]{2}{*}{$\begin{array}{c}\mathrm{V}^{a b s} \\
\left(\mathrm{~km} \mathrm{~s}^{-1}\right)\end{array}$} & \multirow{2}{*}{$\begin{array}{c}\text { 1-gaussian fit } \\
\text { FWHM }^{a b s} \\
(\AA)\end{array}$} & \multirow[b]{2}{*}{$\begin{array}{c}\mathrm{EW}^{a b s} \\
(\AA)\end{array}$} \\
\hline & $\begin{array}{c}\mathrm{V}_{b} \\
\left(\mathrm{~km} \mathrm{~s}^{-1}\right)\end{array}$ & $\begin{array}{c}\mathrm{V}_{r} \\
\left(\mathrm{~km} \mathrm{~s}^{-1}\right)\end{array}$ & $\begin{array}{c}\mathrm{V}^{a b s} \\
\left(\mathrm{~km} \mathrm{~s}^{-1}\right)\end{array}$ & $\begin{array}{c}\mathrm{FWHM}^{a b s} \\
(\AA)\end{array}$ & & & \\
\hline March 31 & $-490 \pm 63$ & $690 \pm 46$ & $122 \pm 37$ & $51 \pm 3$ & $168 \pm 31$ & $63 \pm 3$ & $2.5 \pm 0.2$ \\
\hline April 12 & $-661 \pm 51$ & $494 \pm 41$ & $144 \pm 30$ & $49 \pm 2$ & $186 \pm 21$ & $50 \pm 2$ & $2.6 \pm 0.2$ \\
\hline April 29 & $-656 \pm 27$ & $451 \pm 155$ & $373 \pm 132$ & $36 \pm 11$ & $300 \pm 60$ & $46 \pm 4$ & $1.8 \pm 0.2$ \\
\hline May 25 & - & $718 \pm 21$ & $-120 \pm 48$ & $53 \pm 10$ & $-71 \pm 29$ & $64 \pm 3$ & $2.7 \pm 0.1$ \\
\hline
\end{tabular}

Note. - Columns 2-5 show the results from the 3-gaussian fit: shift of the blue $\left(\mathrm{V}_{b}\right)$ and red $\left(\mathrm{V}_{r}\right)$ emission peaks; shift $\left(\mathrm{V}^{a b s}\right)$, FWHM $\left(\mathrm{FWHM}^{a b s}\right)$ of the absorption component. Columns 6-8 show the results from a 1-gaussian fit after masking the emission core. Column 8 gives the area (i.e. $\mathrm{EW}$ ) of the gaussian. Again the uncertainties were calculated after scaling the error bars to give $\chi_{\nu}^{2}=1$.

TABLE 4

FWZI $^{\mathrm{a}}$ AND Equivalent Widths ${ }^{\mathrm{a}}$ FOR $\mathrm{H} \alpha, \mathrm{H} \beta$, AND Heil $\lambda 4686$

\begin{tabular}{|c|c|c|c|c|c|c|c|}
\hline \multirow[b]{2}{*}{ UT Date } & \multirow{2}{*}{$\overline{\text { FWZI }}$} & \multirow{2}{*}{$\mathrm{H} \alpha$} & \multicolumn{3}{|c|}{$\mathrm{H} \beta^{\mathrm{b}}$} & \multicolumn{2}{|c|}{ — HeII $\lambda 4686$} \\
\hline & & & $\mathrm{FWZI}^{a b s}$ & $\mathrm{FWZI}^{\mathrm{em}}$ & $\mathrm{EW}^{e m}$ & FWZI & EW \\
\hline March 31 & $69 \pm 11$ & $-1.9 \pm 0.2$ & $95 \pm 4$ & 49 & -0.70 & $53 \pm 5$ & $-1.2 \pm 0.2$ \\
\hline April 12 & $63 \pm 2$ & $-1.6 \pm 0.1$ & $98 \pm 5$ & 49 & -0.61 & $69 \pm 19$ & $-0.8 \pm 0.3$ \\
\hline April 29 & $71 \pm 6$ & $-2.1 \pm 0.1$ & $82 \pm 9$ & 54 & -0.95 & $55 \pm 2$ & $-1.4 \pm 0.1$ \\
\hline May 25 & $60 \pm 10$ & $-0.9 \pm 0.2$ & $97 \pm 2$ & 47 & -0.49 & $67 \pm 2$ & $-0.9 \pm 0.2$ \\
\hline
\end{tabular}

${ }^{a}$ In angstroms.

${ }^{b} \mathrm{FWZI}$ of the absorption $\left(\mathrm{FWZI}^{a b s}\right)$ and emission $\left(\mathrm{FWZI}^{e m}\right)$ components of the line profile. $\mathrm{EW}^{\text {em }}$ denotes the equivalent width of the emission component within the broad absorption. Both FWZI ${ }^{e m}$ and $\mathrm{EW}^{e m}$ are lower limits only.

TABLE 5

Line Equivalent Widths ${ }^{\mathrm{a}}$ FOR Hei And Bowen Blend Lines

\begin{tabular}{lcccc}
\hline \hline Date & HeI & HeI & HeI & $\begin{array}{c}\text { Bowen } \\
\text { blend }\end{array}$ \\
UT & 6678 & 5875 & 4921 & - \\
\hline March 31 & $-0.6 \pm 0.1$ & $-0.3 \pm 0.1$ & $-0.2 \pm 0.1$ & - \\
April 12 & $-0.5 \pm 0.1$ & $-0.5 \pm 0.1$ & - & - \\
April 29 & $-0.6 \pm 0.1$ & $-0.3 \pm 0.1$ & $-0.3 \pm 0.1$ & $-0.2 \pm 0.1$ \\
May 25 & $-0.3 \pm 0.1$ & $-0.3 \pm 0.1$ & - & - \\
\hline
\end{tabular}

Note. - Only the equivalent widths for the stronger lines were measured.

${ }^{a}$ In angstroms. 